\title{
G Research Square $=1$ \\ Effect of Previous Chlamydia Trachomatis Infection on the Outcomes of IVF/ICSI Treatment: A retrospective study
}

\section{Dan Zhang}

Sun Yat-sen University First Affiliated Hospital

\section{Zengyan Wang}

Sun Yat-sen University First Affiliated Hospital

\section{Xingyuan Hu}

Sun Yat-sen University Zhongshan School of Medicine

\section{Chunguang Ma}

Sun Yat-sen University First Affiliated Hospital

\section{Yuanlin Ma}

Sun Yat-sen University First Affiliated Hospital

\section{Yanwen Xu ( $\nabla$ xuyanwen@mail.sysu.edu.cn )}

Reproductive Medical Center, the First Affiliated Hospital of Sun Yat-sen https://orcid.org/0000-00016834-7009

\section{Research Article}

Keywords: Chlamydia trachomatis infection, In vitro fertilization, Intracytoplasmic sperm injection, Pregnancy outcome

Posted Date: February 28th, 2022

DOI: https://doi.org/10.21203/rs.3.rs-885642/v1

License: (c) (1) This work is licensed under a Creative Commons Attribution 4.0 International License. Read Full License

Version of Record: A version of this preprint was published at BMC Pregnancy and Childbirth on April 10th, 2022. See the published version at https://doi.org/10.1186/s12884-022-04624-z. 


\section{Abstract}

Background: The impact of Chlamydia trachomatis infection (CT) on the outcomes of In-Vitro Fertilizition / Intracytoplasmic sperm injection (IVF/ICSI) has been controversial.

Methods: This retrospective study investigated the effect of cured CT infection and the intervals between infection and the first embryo transfer on pregnancy outcomes in infertile patients undergoing IVF/ICSI. A total of 431 infertility women aged 20-38 years with or without Chlamydia trachomatis infection before fresh/ frozen embryo transfer were included. We subdivided the infected group into two subgroups for $\leq$ 3 months and $>3$ months according to the different intervals between Chlamydia trachomatis positive testing and embryo transfer. The effect of chlamydia infection and the intervals between infection and embryo transfer on pregnancy outcomes was analyzed.

Results: Our results revealed that implantation rate was significantly lower and the premature rupture of membranes (PROM) was higher in women with CT infection than non-infection. The multivariate logistic regression analysis adjusting for baseline characteristics showed no significant difference in embryo implantation between neither two groups nor two subgroups while patients in infection group have higher rate of PROM than those in control group.

Conclusions: The study suggests that previous Chlamydia trachomatis infection would not impair embryo implantation but lead to high risk of PROM and the intervals between infection and embryo transfer would not impact the pregnancy outcomes of IVF/ICSI.

\section{Background}

Genital chlamydia trachomatis (CT) infection is one of the most common sexually transmitted diseases throughout the world. Presenting as asymptomatic in most cases [1], chlamydial infections can cause infection for more than one year among up to $50 \%$ of the infected people if not treated in time [2], which can lead to a series of serious complications such as pelvic inflammatory disease [3], ectopic pregnancy, fecundity decline and even tubal obstruction $[4,5]$.

Since the infection of CT has shown adverse impact on human reproductive capability, however, the impact of CT infection on IVF/ICSI among infertile patients has been controversial and previous studies have reached different conclusions. It was reported that $\mathrm{CT}$ affected not only the fallopian tubes which can lead to tubal factor infertility, but also other genital tract sites [6-9]. The presence of anti-Chlamydia antibodies or heat shock protein 60 (HSP 60) may contribute to autoimmune response and the persistent subclinical chlamydial infection, leading to adverse effects on pregnancy outcomes in IVF/ICSI cycles [810]. It has been suggested that abnormal immune induced by persistent CT infection might promote tubal damage and have impact on IVF/ICSI outcomes. On the other hand, some studies found that there was no significant difference in outcomes of IVF/ICSI between patients with CT infection and those without infection [11-14]. 
Furthermore, there were few reports on the optimal time for embryo transfer after cure of chlamydia infection. As a result, we conducted a retrospective cohort study to further explore the impact of CT infection and the intervals between infection and embryo transfer on IVF / ICSI outcomes.

\section{Methods}

\section{Study population}

In this retrospective study, all women had screened for Mycoplasma genitalium, Chlamydia, bacterial vaginosis and trichomonas vaginalis before entering IVF/ICSI. Women who tested CT positive before oocyte retrieval or frozen embryo transfer of IVF/ICSI cycles were included in the experimental group when the CT test turned negative after one standard treatment. Women who tested CT negative without female tubal factors were enrolled as the control group from June 2018 to January 2020 of the First Affiliated Hospital of Sun Yat-sen University. The timeline of diagnosis and IVC/ICSI stage of infected group shown in Figure1. The other inclusive criteria: (1) undergoing an IVF / ICSI program. (2) Age between 20 and 38 years. (3) BMI $\leq 28 \mathrm{~kg} / \mathrm{m} 2$. (4) Normal ovarian reserve (bFSH < 10lU/L). (5) Number of oocytes retrieved $\geq 5$. (6) Total number of oocytes retrieval cycles $\leq 3$. (7) Endometrial thickness on hCG day or progesterone conversion day $\geq 7 \mathrm{~mm}$. The exclusion criteria: (1) Patients with intrauterine abnormalities, including: endometrial polyps or submucosal fibroids, history of uterine adhesiolysis, uterine malformation. (2) Patients with a history of recurrent spontaneous abortion (RSA), RSA was defined as three or more consecutive pregnancies loss (including biochemical pregnancy abortion and clinical pregnancy loss). (3) Three or more times of embryo transfer without clinical pregnancy. (4) Patients with hydrosalpinx. (5) Patients with uncontrolled hyperprolactinemia, hypothyroidism and severe medical or surgical diseases. The institutional ethical board of the First Affiliated Hospital of Sun Yat-sen University approved this retrospective study.

\section{Fig. 1 Timeline of Diagnosis and IVC/ICSI Stage of the Infected Group}

\section{CT Detection and Treatments}

All patients were required to detect CT before oocyte retrieval or frozen embryo transfer every 6 months in IVF/ICSI cycles. The specimens were cervical swabs detected chlamydial lipopolysaccharide (LPS) by enzyme immunoassay (EIA). Patients tested positive were treated with a 7-day regimen of azithromycin or doxycycline before IVF/ICSI according to the recommendations of the Center for Disease Control and Prevention [15]..Sexual partners of CT-positive patients were treated at the same time. Only when patients retested negative after completing therapy can they enter IVF/ICSI-ET or frozen embryo transfer cycles.

\section{Ovarian stimulation}


Patients received the luteal-phase gonadotrophin-releasing hormone agonist or gonadotrophin antagonist protocols. Since the pituitary was adequately suppressed, or on the 2-3 day of menstruation, ovarian stimulation was induced with human menopausal gonadotrophin and recombinant FSH (Merck Serono) individualized. Human chorionic gonadotrophin (hCG) was injected when two or more dominant follicles reached $18 \mathrm{~mm}$ in diameter. Then we collected oocytes 36 hours after hCG injection.

\section{Outcome measures}

An infertility investigation was performed in all couples. We collected and analyzed data of general characteristics, ovulation induction, embryonic development and pregnancy outcomes. The primary outcome was live-birth rates, the secondary outcomes were the embryo implantation rate, chemical pregnancy rate, ectopic pregnancy rate, abortion rate and clinical pregnancy rate.

In order to investigate whether the interval between CT infection and embryo transfer had any effective on pregnancy outcomes, we further divided the infection group into two subgroups: $\leq 3$ months and $>3$ months according to the intervals between CT infection and embryo transfer. Data of two subgroups were also compared.

\section{Statistical analysis}

The statistical analyses were performed by SPSS 24.0 software. Pearson's Chi square test was used to analyze differences between relative frequencies. Student's t-test and one-way ANOVA were used to compare continuous variables. $\mathrm{P}<0.05$ was considered statistically significant. The multivariate logistic regression model was fitted to analyze the effect of $\mathrm{CT}$ infection and the intervals between $\mathrm{CT}$ infection

and embryo transfer on live birth adjusting for the study variables (baseline characteristics, protocols, and transfer cycles) for live birth rate. Odds ratios or hazard ratios and $95 \%$ confidence intervals were calculated. A two-sided a level of 0.05 was considered as statistically significant.

\section{Results}

\section{Baseline Characteristics}

From June 2018 to January 2020, a total of 690 patients were tested positive for C. trachomatis during the inclusion period, of whom 129 couples were eligible and among 687 patients tested CT negative and undergoing ICSI only for male infertility, 302 were enrolled for this analysis (Fig. 1).

\section{Fig. 2 The recruitment process}

In the infection group, 88 couples underwent IVF, 37 underwent ICSI and 4 underwent IVF+ICSI. The fresh cycle of 74 couples in the infection group and 151 in the control group were cancelled and they 
underwent thawed embryo transfer (TET) later mainly for ovarian hyperstimulation syndrome (OHSS), elevated progesterone and endometrial factors.

Cycle baseline characteristics were similar between CT positive and negative group, such as age, BMI, infertility duration and basic $\mathrm{FSH}$, while the incidence of secondary infertility and previous ectopic pregnancy rate was higher in CT infection group than that in control group (Table.1).

\section{Table.1 Baseline Characteristics of the infection and control group}

\begin{tabular}{|llll|}
\hline Indexes & $\begin{array}{l}\text { Infection group } \\
(\mathbf{n = 1 2 9 )}\end{array}$ & $\begin{array}{l}\text { Control group } \\
(\mathbf{n}=\mathbf{3 0 2})\end{array}$ & $\mathbf{P}$ \\
\hline Age (years) & $30.82 \pm 3.80$ & $30.85 \pm 3.65$ & 0.939 \\
\hline $\mathrm{BMI}(\mathrm{kg} / \mathrm{m} 2)$ & $21.19 \pm 2.25$ & $21.20 \pm 2.34$ & 0.962 \\
\hline Infertility duration (years) & $3.40 \pm 2.05$ & $3.73 \pm 2.56$ & 0.208 \\
\hline Basal FSH (IU/L) & $5.35 \pm 1.38$ & $5.52 \pm 1.40$ & 0.237 \\
\hline Types of infertility (\%) & & & $0.028^{*}$ \\
\hline Primary infertility & $48.8(63 / 129)$ & $60.3(182 / 302)$ & \\
\hline Secondary infertility & $51.2(66 / 129)$ & $39.7(120 / 302)$ & \\
\hline Previous ectopic pregnancy (\%) & $16.27(21 / 129)$ & $1.98(6 / 302)$ & $<0.001^{*}$ \\
\hline Continuous variables are presented as the mean \pm SD & \\
\hline
\end{tabular}

There were no significant differences between two groups concerning initial dose of gonadotropins (Gn), days of $G n$ used, total dose of $G n$, level of serum $E_{2}$ and endometrial thickness on the day of $h C G$ injection, number of retrieved oocytes, blastocyst formation rate and type of embryo transfer, but infection group had lower good-quality embryo rate and embryo transfer numbers, higher blastocyst transfer rate (Table.2).

Table.2 Parameters of Patients with and without C. trachomatis Infection 


\begin{tabular}{|c|c|c|c|}
\hline Indexes & $\begin{array}{l}\text { Infection group } \\
(n=129)\end{array}$ & $\begin{array}{l}\text { Control group } \\
(n=302)\end{array}$ & $\mathbf{P}$ \\
\hline Initial dose of Gn (IU) & $210.48 \pm 58.38$ & $201.44 \pm 55.42$ & 0.175 \\
\hline Days of Gn used (IU) & $10.69 \pm 2.39$ & $10.07 \pm 1.73$ & 0.060 \\
\hline Total dose of Gn (IU) & $2279.70 \pm 961.29$ & $2086.76 \pm 800.81$ & 0.054 \\
\hline level of $E_{2}$ on hCG day $(\mathrm{ng} / \mathrm{ml})$ & $3203.27 \pm 1244.91$ & $2986.53 \pm 1271.71$ & 0.157 \\
\hline Endometrial thickness (mm) & $11.70 \pm 2.20$ & $11.79 \pm 3.15$ & 0.804 \\
\hline Numbers of oocytes retrieved & $15.80 \pm 6.96$ & $15.79 \pm 6.96$ & 0.990 \\
\hline Good-quality embryo rate (\%) & $83.3(649 / 779)$ & $86.5(1438 / 1662)$ & $0.041^{*}$ \\
\hline Blastocyst formation on Day 5 or $6(\%)$ & $63.3(584 / 922)$ & 61.2(1217/1987) & 0.282 \\
\hline Transferred embryo stage (\%) & & & $0.006^{\star}$ \\
\hline D3 & $53.5(69 / 129)$ & $67.5(204 / 302)$ & \\
\hline D5/6 & $46.5(60 / 129)$ & $32.5(98 / 302)$ & \\
\hline Type of embryo transfer (\%) & & & 0.161 \\
\hline TET & $57.4(74 / 129)$ & $50(151 / 302)$ & \\
\hline Fresh cycle & $42.6(55 / 129)$ & $50(151 / 302)$ & \\
\hline Number of transferred embryos (\%) & & & $0.003^{*}$ \\
\hline 1 & $51.9(67 / 129)$ & $36.4(110 / 302)$ & \\
\hline 2 & $48.1(62 / 129)$ & $63.6(192 / 302)$ & \\
\hline
\end{tabular}

\section{Pregnancy outcomes}

The implantation rate $(28,2 \%$ vs $36.2 \%, P=0.046)$ and multiple pregnancies rate $(9.8 \%$ vs $26.8 \%, P=0.013)$ were found significantly lower and the PROM rate $(7.8 \%$ vs $0.7 \%, P=0.025)$ was higher in CT infection group comparing with the $\mathrm{CT}$ negative. No significant difference was found in the chemical pregnancy rate $(48.1 \%$ vs $53.0 \%, P=0.349)$, abortion rate $(21.6 \%$ vs $12.7 \%, P=0.127)$, clinical pregnancy rate $(39.5 \%$ vs $47.0 \%, P=0.152)$, and ectopic pregnancy rate $(2.0 \%$ vs $1.3 \%, P=0.709)$ between the two groups (Table.3).

During pregnancy, seven patients developed hypertensive disorders of pregnancy, one developed hyperthyroidism in pregnancy, one suffered with placenta previa and one had low amniotic fluid in the control group, which are not statistically significant comparing to the infection group. The rate of gestational diabetes mellitus is slightly higher in the control group than the infection group. 
As for conditions of birth and delivery, the rates of abortion, live birth and premature birth and the average birth weight of newborn are similar in both groups. One patient in the control group was diagnosed pulmonary embolism in the postpartum period. There was one neonate with cryptorchidism, one with strephenopodia and one with gastroschisis in the control group and one in the infection group was born with congenital heart diseases.

\section{Table.3 Clinical Outcomes of Patients with and without C. trachomatis Infection}

\begin{tabular}{|llll|}
\hline Indexes & Infection group (n=129) & $\begin{array}{l}\text { Control group } \\
(\mathbf{n = 3 0 2})\end{array}$ & $\mathbf{P}$ \\
\hline Implantation rate (\%) & & $36.2(177 / 489)$ & $0.046^{\star}$ \\
\hline Chemical pregnancy rate (\%) & $48.1(62 / 129)$ & $53.0(160 / 302)$ & 0.349 \\
\hline Clinical pregnancy rate (\%) & $39.5(51 / 129)$ & $47.0(142 / 302)$ & 0.152 \\
\hline Ectopic pregnancy rate (\%) & $2.0(1 / 51)$ & $1.3(2 / 160)$ & 0.709 \\
\hline Multiple pregnancies rate (\%) & $9.8(5 / 51)$ & $26.8(38 / 142)$ & $0.013^{\star}$ \\
\hline Gestational diabetes mellitus (\%) & $0.0(0 / 51)$ & $9.9(14 / 142)$ & $0.044^{\star}$ \\
\hline Hypertensive disorders of pregnancy (\%) & $0.0(0 / 51)$ & $4.9(7 / 142)$ & 0.239 \\
\hline Hyperthyroidism in pregnancy (\%) & $0.0(0 / 51)$ & $0.7(1 / 142)$ & 1.000 \\
\hline Placenta previa (\%) & $0.0(0 / 51)$ & $0.7(1 / 142)$ & 1.000 \\
\hline Premature rupture of membranes (\%) & $7.8(4 / 51)$ & $0.7(1 / 142)$ & $0.025^{\star}$ \\
\hline Oligohydramnios (\%) & $0.0(0 / 51)$ & $0.7(1 / 142)$ & 1.000 \\
\hline Abortion rate (\%) & $21.6(11 / 51)$ & $12.7(18 / 142)$ & 0.127 \\
\hline Live birth rate (\%) & $30.2(39 / 129)$ & $40.1(121 / 302)$ & 0.053 \\
\hline Gestational age (w) & $38.3 \pm 1.9$ & $38.1 \pm 2.1$ & 0.670 \\
\hline Premature birth rate (\%) & $20.5(8 / 39)$ & $21.7(26 / 120)$ & 0.879 \\
\hline Pulmonary embolism (\%) & $0.0(0 / 39)$ & $0.8(1 / 120)$ & 1.000 \\
\hline
\end{tabular}

The univariate logistic regression showed live birth rate was associated with age, BMI, infertility duration, basal $F S H$, initial dose of $G$, total dose of $G n$, level of $E_{2}$, approach of fertilization and whether $C T$ infection or not. After adjusting for these factors, significant difference was still found in the rate of premature rupture of membranes between two groups, while no difference was showed comparing other pregnancy outcomes. (Table.4).

\section{Table.4 Clinical Outcomes after Adjustment}




\begin{tabular}{|lllll|}
\hline Indexes & OR $(95 \% \mathrm{Cl})$ & $\mathbf{P}$ & Adjusted OR (95\%Cl) & P \\
\hline $\begin{array}{l}\text { Chemical pregnancy rate } \\
(\%)\end{array}$ & $0.821(0.544,1.241)$ & 0.350 & $1.058(0.486,2.302)$ & 0.887 \\
\hline Clinical pregnancy rate (\%) & $0.737(0.485,1.120)$ & 0.153 & $0.932(0.434,1.999)$ & 0.856 \\
\hline Abortion rate (\%) & $1.894(0.826,4.347)$ & 0.132 & $0.703(0.121,4.082)$ & 0.695 \\
\hline Live birth rate (\%) & $0.648(0.417,1.007)$ & 0.054 & $0.897(0.411,1.958)$ & 0.786 \\
\hline $\begin{array}{l}\text { Multiple pregnancies rate } \\
(\%)\end{array}$ & $0.297(0.110,0.805)$ & 0.017 & $0.174(0.21,1.474)$ & 0.109 \\
\hline $\begin{array}{l}\text { Premature rupture of } \\
\text { membranes (\%) }\end{array}$ & $12.000(1.308,110.051)$ & 0.028 & $206.675(5.103,8369.836)$ & 0.005 \\
\hline $\begin{array}{l}\text { Categorical variables are presented as percentage (their frequencies), OR and 95\% Cl. Chemical } \\
\text { pregnancy rate, clinical pregnancy rate, abortion rate and live birth rate are additionally adjusted for } \\
\text { age, BMI, bFSH and infertility factors, approach of fertilization, transferred embryo stage (D3, D5 or } \\
\text { D6), Number of transferred embryos. Italic values represents statistical significance. }\end{array}$ & \\
\hline
\end{tabular}

\section{The pregnancy outcomes of different intervals between infection and embryo transfer}

According to previous study (Patton et al.,1994), the detection of antibody against CT may associate with the intervals from the end of treatment. Based on the findings above and the clinical experience, we further subdivided the infection group into two subgroups for $\leq 3$ months and $>3$ months, according to the different intervals between CT infection and embryo transfer. All patients in CT positive group were treated with a 7-days regimen and retested CT negative before transferring embryos. The characteristics and outcomes of the two subgroups were also compared (Table.5).

Table.5 Characteristics and outcomes after IVF/ICSI-ET in the infected group 


\begin{tabular}{|c|c|c|c|}
\hline Indexes & $\begin{array}{l}\leq 3 \text { months } \\
(n=60)\end{array}$ & $\begin{array}{l}>3 \text { months } \\
(n=69)\end{array}$ & $\mathbf{P}$ \\
\hline Age (years) & $31.35 \pm 3.89$ & $30.95 \pm 3.82$ & 0.263 \\
\hline $\mathrm{BMI}(\mathrm{kg} / \mathrm{m} 2)$ & $21.32 \pm 2.31$ & $21.01 \pm 2.39$ & 0.455 \\
\hline Basal FSH (U/L) & $5.48 \pm 1.44$ & $5.26 \pm 1.34$ & 0.385 \\
\hline Types of infertility (\%) & & & 0.061 \\
\hline Primary infertility & $40(24 / 60)$ & $56.5(39 / 69)$ & \\
\hline Secondary infertility & $60(36 / 60)$ & $43.5(30 / 69)$ & \\
\hline Endometrial thickness (mm) & $11.71 \pm 2.21$ & $11.60 \pm 2.36$ & 0.789 \\
\hline Approach of Fertilization (\%) & & & 0.979 \\
\hline IVF & $68.3(41 / 60)$ & $68.1(47 / 69)$ & \\
\hline ICSI/IVF+ICSI & $31.7(19 / 60)$ & $31.9(22 / 69)$ & \\
\hline Type of embryo transfer (\%) & & & 0.053 \\
\hline TET & $48.3(29 / 60)$ & $65.2(45 / 69)$ & \\
\hline Fresh cycle & $51.7(31 / 60)$ & $34.8(24 / 69)$ & \\
\hline Number of transferred embryos (\%) & & & 0.767 \\
\hline 1 & $53.3(32 / 60)$ & $50.7(35 / 69)$ & \\
\hline 2 & $46.7(28 / 60)$ & $49.3(34 / 69)$ & \\
\hline Transferred embryo stage (\%) & & & 0.500 \\
\hline D3 & $56.7(34 / 60)$ & $50.7(35 / 69)$ & \\
\hline $\mathrm{D} 5 / 6$ & $43.3(26 / 60)$ & $49.3(34 / 69)$ & \\
\hline Good-quality embryo rate (\%) & $86.7(284 / 328)$ & $80.9(365 / 451)$ & $0.034^{*}$ \\
\hline Blastocyst formation rate (\%) & $59.9(240 / 400)$ & $65.9(344 / 522)$ & 0.064 \\
\hline Chemical pregnancy rate (\%) & $38.3(23 / 60)$ & $50.7(35 / 69)$ & 0.158 \\
\hline Clinical pregnancy rate (\%) & $36.7(22 / 60)$ & $42.0(29 / 69)$ & 0.534 \\
\hline Multiple pregnancies rate (\%) & $0(0 / 22)$ & $17.2(5 / 29)$ & 0.115 \\
\hline Premature rupture of membranes (\%) & $4.5(1 / 22)$ & $13.6(3 / 22)$ & 0.600 \\
\hline Abortion rate $(\%)$ & $22.7(5 / 22)$ & $20.7(6 / 29)$ & 1.000 \\
\hline Live birth rate (\%) & $28.3(17 / 60)$ & $31.9(22 / 69)$ & 0.661 \\
\hline
\end{tabular}




\begin{tabular}{|llll|}
\hline Indexes & $\begin{array}{l}\mathbf{\leq 3} \text { months } \\
(\mathbf{n = 6 0})\end{array}$ & $\begin{array}{l}>3 \text { months } \\
(\mathbf{n = 6 9 )}\end{array}$ & $\mathbf{P}$ \\
\hline Gestational age(w) & $37.4 \pm 3.5$ & $38.2 \pm 2.4$ & 0.417 \\
\hline Premature birth rate (\%) & $13.6(3 / 22)$ & $17.9(5 / 28)$ & 0.988 \\
\hline Birth weight (g) & 3082.9 & 2844.8 & 0.271 \\
\hline Macrosomia (\%) & $4.5(1 / 22)$ & $3.6(1 / 28)$ & 1.000 \\
\hline Normal birth weight infant (\%) & $81.8(18 / 22)$ & $75.0(21 / 28)$ & 0.815 \\
\hline Low birth weight infant (\%) & $13.6(3 / 22)$ & $10.7(3 / 28)$ & 1.000 \\
\hline Very low birth weight infant (\%) & $0(0 / 22)$ & $10.7(3 / 28)$ & 0.325 \\
\hline Congenital malformation (\%) & $4.5(1 / 22)$ & $0(0 / 28)$ & 0.440 \\
\hline
\end{tabular}

The baseline characteristics were similar and comparable. Patients with interval $>3$ months showed slightly lower good-quality embryo rate than those $\leq 3$ months. Except for this, there were no significant differences in pregnancy outcomes between the subgroups.

The univariate logistic regression showed live birth rate was associated with age, BMI, infertility duration, basal $\mathrm{FSH}$, initial dose of $\mathrm{Gn}$, total dose of $\mathrm{Gn}$, level of $\mathrm{E}_{2}$, approach of fertilization and whether $\mathrm{CT}$ infection or not.

The multivariate logistic regression showed the live the birth rate can be affected by age, BMI, infertility duration, basal $\mathrm{FSH}$, initial dose of $\mathrm{Gn}$, total dose of $\mathrm{Gn}$, level of $\mathrm{E}_{2}$, approach of fertilization and whether CT infection or not, which is consistent with the findings above. No significant difference was found in the clinical outcomes after adjustment (Table.6).

Table.6 Clinical Outcomes after Adjustment in the infected group

\begin{tabular}{|lllll|}
\hline Indexes & OR $(95 \% \mathrm{Cl})$ & P & Adjusted OR (95\%Cl) & P \\
\hline Chemical pregnancy rate (\%) & $1.656(0.820,3.343)$ & 0.158 & $1.533(0.703,3.343)$ & 0.282 \\
\hline Clinical pregnancy rate (\%) & $1.286(0.624,2.684)$ & 0.495 & $1.140(0.519,2.502)$ & 0.745 \\
\hline Abortion rate (\%) & $0.862(0.168,2.772)$ & 0.593 & $0.555(0.121,2.541)$ & 0.448 \\
\hline Live birth rate (\%) & $1.313(0.603,2.856)$ & 0.493 & $1.184(0.531,2.731)$ & 0.692 \\
\hline $\begin{array}{l}\text { Categorical variables are presented as percentage (their frequencies), OR and 95\% Cl. Chemical } \\
\text { pregnancy rate, clinical pregnancy rate, abortion rate and live birth rate are additionally adjusted for } \\
\text { age, BMI, bFSH and infertility factors, approach of fertilization, transferred embryo stage (D3, D5 or } \\
\text { D6), number of transferred embryos. }\end{array}$ \\
\hline
\end{tabular}

\section{Discussion}


As we all know, the ICSI cycles have similar pregnancy outcomes to the IVF cycles [21]. In this study, women who underwent ICSI-ET treatment without fallopian tube factors were included in our control group, so as to minimize the impact of fallopian tube problems probably caused by previous CT infection on the outcome of the control group. The risk of major birth defects after ICSI were not found in the control group [22]. The infection and control groups were comparable regarding demographic data, infertility duration, duration of stimulation, duration of treatment, $\mathrm{E}_{2}$ level and endometrial thickness on the day of HCG administration. There was no statistically significant difference observed between the two groups regarding the mean number of oocytes retrieved per patient and blastocyst formation rate, but the mean number of embryos transferred per patient and good quality embryos rate were significantly lower while the rate of premature rupture of membranes was higher in infection group than in control group.

It had suggested that adverse reproductive sequelae of ectopic pregnancy due to CT infection had been associated with antibody response to the chlamydial heat shock protein 60 (CHSP 60) $[4,5,16]$. In our infection group, infertile women combined with tubal factors had higher incidence of previous ectopic pregnancy than the control group without tubal factors. However, during our IVF/ICSI-ET treatment, CT infection did not increase the incidence of ectopic pregnancy significantly. We speculated the possible reason was that previous studies on the impact of CT on natural conception did not address whether CT is prescriptively treated and turned negative before pregnancy, and some are even found to be CT positive during pregnancy. While in this study, the patients started the treatment for IVF/ICSI-ET only when CT was prescriptive therapy and the cervical swab resulted negative for Chlamydia.

The impacts of CT on the pregnancy outcomes in assisted reproductive technology (ART) are still controversial. Several researchers demonstrated negative effects of previous chlamydial infection. According to Witkin et al. [10], the prevalence of CHSP 60 antibodies induce an inflammatory reaction in the uterus that may impair embryo implantation. Pacchiarotti et al. [9] found the presence of antichlamydia antibodies in both the serum and follicular fluid reduced the number of mature oocytes in antibodies-positive group, leading to the poor IVF outcome. On the contrary, some studies demonstrated that previous CT infection does not affect pregnancy outcomes in IVF/ICSI (11-13,17). Claman et.al [18] showed no differences in pregnancy rates or outcomes in patients with and without serologic evidence of previous CT infection. Nevertheless, Serum antichlamydial antibodies do not correlate as well with cervical infection. When infected by CT in genital tract, the expression and distribution of different kind of antibodies are not synchronoused [7]. Therefore, the misclassification of chlamydia stages may account for the different impacts of CT on pregnancy outcomes.

A global systematic review [19] demonstrates associations between chlamydia and several adverse pregnancy outcomes. But there are some limitations due to the substantial heterogeneity across studies and the confounding variables such as medical history. The possible impacts of the related factors on the results cannot be explained when quantifying the correlation. In addition, differences of study population, sample size, treatment and random error may also have certain impact on the result. Our results of multivariable regression analysis showed that CT infection was not significantly associated 
with lower chemical pregnancy rate, clinical pregnancy rate, abortion rate and live birth rate, which confirmed that previous CT infection is not a risk factor of adverse pregnant outcomes of IVF/ICSI.

The rate of premature rupture of membranes was higher in infection group than in control group. The persistent subclinical chlamydial infection and the abnormal immune induced by previous CT infection may increase the concentration of polymorphonuclear leukocytes which is capable of altering the local tissue $\mathrm{pH}$ and thus perturbing lysosomal membranes in the adjacent chorioamnion and histological chorioamnionitis. The effect could lead to weaken membranes and hence membrane rupture $[23,24]$. According to previous study [20], the detection of antibody against CT may associate with the intervals from the end of treatment. Based on the findings above and the clinical experience, we further subdivided the infection group into two subgroups for $\leq 3$ months and $>3$ months. There was no differences between the subgroups, showing that the group $>3$ months interval between CT infection diagnosis and embryo transfer have similar outcomes in the IVF/ICSI to those $\leq 3$ months. According to Hoenderboom et.al. [14], Women with previous CT infection have similar overall pregnancy rates as those tested negative but took longer to get pregnant possibly because of intratubal microdamage. Therefore, we suggested that the infection of CT without hydrosalpinx will not impair the ability of embryos implantation and development after their transfer to the uterus. However, the PROM rate showed higher in the infection group than the control group while no different was found between the subgroups. It suggested that longer time interval would not reduce the impact of CT infection on the risk of PROM.

In this study, we formulated strict inclusion criteria, exclusion criteria and perform a multivariable logistic regression, which is conducive to eliminate the possible effects of general characteristics in the transplantation cycles. Compared with previous studies, we greatly controlled the possible confounders between the infection group and the control group. Subgroup stratification was performed in the infected group to analyze whether the time intervals between infection and embryo transfer had any effect on pregnancy outcomes. The results showed that prolonged interval did not increase implantation rate, clinical pregnancy rate, live birth rate, and did not reduce miscarriage rate when the interval $>3$ months verse $\leq 3$ months. Our results suggest that there is no need to delay embryo transfer after cure of CT infection during the IVF/ICSI cycle.

\section{Conclusion}

This study demonstrates that cured CT infection without hydrosalpinx will not impair embryo implantation, clinical pregnancy and live birth but lead women at high risk of PROM during IVF/ICSI. Although CT infection does not significantly affect the live birth rate, it can result in an increased incidence of PROM, while extended interval between infection and embryo transfer will not decrease the risk of adverse outcomes, which may provide a good warning for clinical practice. There are still many deficiencies due to the limited sample size, testing technology and other reasons. We have not analyzed the long-term effects of previous CT infection to the infants. The effects of longer or shorter time intervals on pregnancy outcomes are unknown yet. Further study is warranted to confirm our proposal. 


\section{Abbreviations}

IVF: In-Vitro Fertilization;

ICSI: Intracytoplasmic sperm injection;

Gn: Gonadotropins;

CT: Chlamydia trachomatis;

HSP 60: Heat shock protein 60;

CHSP 60: Chlamydial heat shock protein 60

\section{Declarations}

\section{Ethics approval and consent to participate}

This study was performed under the approval of the local ethics committee of the First Affiliated Hospital of Sun Yat-Sen University and all patients provided signed written informed consent.

\section{Consent for publication}

Not Applicable.

\section{Availability of data and materials}

The datasets analyzed during the current study are not publicly available given the privacy of patient data and we plan to further analyze the data to dig up much more clinic information. But they are available from the corresponding author on reasonable request.

\section{Competing interests}

The authors declare that they have no competing interests.

\section{Funding}

This work was supported by Guangdong Provincial Key Laboratory of Reproductive Medicine. (Grant numbers 2012A061400003)

\section{Authors' contributions}

(I) Conception and design: YX, DZ; (II) Administrative support: YX; (III) Provision of study materials or patients: DZ, ZW; (IV) Collection and assembly of data: DZ, ZW, CM; (V) Data analysis and interpretation: $\mathrm{DZ}, \mathrm{XH}, \mathrm{YM}$; (VI) Manuscript writing: DZ, XH; (VII) Final approval of manuscript: All authors. 
Acknowledgements

Not applicable.

\section{References}

1. Low N. Screening programmes for chlamydial infection: when will we ever learn? BMJ. 2007;334(7596):725-8.

2. Geisler WM. Duration of untreated, uncomplicated Chlamydia trachomatis genital infection and factors associated with chlamydia resolution: a review of human studies. J Infect Dis. 2010;201(Suppl 2):104-13.

3. Davies B, Turner KME, Benfield T, et al. Pelvic inflammatory disease risk following negative results from chlamydia nucleic acid amplification tests (NAATs) versus non-NAATs in Denmark: A retrospective cohort. PLoS Med. 2018;15(1):e1002483.

4. Menon S, Timms P, Allan JA, et al. Human and Pathogen Factors Associated with Chlamydia trachomatis-Related Infertility in Women. Clin Microbiol Rev. 2015;28(4):969-85.

5. Davies B, Turner KME, Frølund M, et al. Risk of reproductive complications following chlamydia testing: a population-based retrospective cohort study in Denmark. Lancet Infect Dis. 2016;16(9):1057-64.

6. Keay SD, Barlow R, Eley A, Masson GM, Anthony FW, Jenkins JM. The relation between immunoglobulin $\mathrm{G}$ antibodies to Chlamydia trachomatis and poor ovarian response to gonadotropin stimulation before in vitro fertilization. Fertil Steril. 1998;70(2):214-8.

7. Neuer A, Lam KN, Tiller FW, Kiesel L, Witkin SS. Humoral immune response to membrane components of Chlamydia trachomatis and expression of human $60 \mathrm{kDa}$ heat shock protein in follicular fluid of in-vitro fertilization patients. Hum Reprod. 1997;12(5):925-9.

8. Jakus S, Neuer A, Dieterle S, Bongiovanni AM, Witkin SS. Antibody to the Chlamydia trachomatis 60 $\mathrm{kDa}$ heat shock protein in follicular fluid and in vitro fertilization outcome. Am J Reprod Immunol. 2008;59(2):85-9.

9. Pacchiarotti A, Sbracia M, Mohamed MA, et al. Autoimmune response to Chlamydia trachomatis infection and in vitro fertilization outcome. Fertil Steril. 2009;91(3):946-8.

10. Witkin SS, Sultan KM, Neal GS, Jeremias J, Grifo JA, Rosenwaks Z. Unsuspected Chlamydia trachomatis infection and in vitro fertilization outcome. Am J Obstet Gynecol. 1994;171(5):1208-14.

11. Spandorfer SD, Neuer A, LaVerda D, et al. Previously undetected Chlamydia trachomatis infection, immunity to heat shock proteins and tubal occlusion in women undergoing in-vitro fertilization. Hum Reprod. 1999;14(1):60-4.

12. Xie QZ, Xu WM, Qi QR, Luo ZL, Dong L. Patients with cervical Ureaplasma Urealyticum and Chlamydia Trachomatis infection undergoing IVF/ICSI-ET: The need for new paradigm. J Huazhong Univ Sci Technolog Med Sci. 2016;36(5):716-22. 
13. Zhu Y, Yin B, Wu T, et al. Comparative study in infertile couples with and without Chlamydia trachomatis genital infection. Reprod Health. 2017;14(1):5.

14. Hoenderboom BM, van Bergen JEAM, Dukers-Muijrers NHTM, et al. Pregnancies and Time to Pregnancy in Women With and Without a Previous Chlamydia trachomatis Infection. Sex Transm Dis. 2020;47(11):739-47.

15. Workowski KA, Bolan GA. Centers for Disease Control and Prevention. Sexually transmitted diseases treatment guidelines, 2015 [published correction appears in MMWR Recomm Rep. 2015 Aug 28;64(33):924]. MMWR Recomm Rep. 2015;64(RR-03):1-137.

16. Brunham RC, Peeling R, Maclean I, Kosseim ML, Paraskevas M. Chlamydia trachomatis-associated ectopic pregnancy: serologic and histologic correlates. J Infect Dis. 1992;165(6):1076-81.

17. Gaudoin M, Rekha P, Morris A, Lynch J, Acharya U. Bacterial vaginosis and past chlamydial infection are strongly and independently associated with tubal infertility but do not affect in vitro fertilization success rates. Fertil Steril. 1999 Oct;72(4):730-2.

18. Claman P, Amimi MN, Peeling RW, Toye B, Jessamine P. Does serologic evidence of remote Chlamydia trachomatis infection and its heat shock protein (CHSP 60) affect in vitro fertilizationembryo transfer outcome? Fertil Steril. 1996;65(1):146-9.

19. Tang W, Mao J, Li KT, et al. Pregnancy and fertility-related adverse outcomes associated with Chlamydia trachomatis infection: a global systematic review and meta-analysis. Sex Transm Infect. 2020;96(5):322-9.

20. Patton DL, Askienazy-Elbhar M, Henry-Suchet $J$, et al. Detection of Chlamydia trachomatis in fallopian tube tissue in women with postinfectious tubal infertility. Am J Obstet Gynecol. 1994;171(1):95-101.

21. Calderón G, Belil I, Aran B, et al. Intracytoplasmic sperm injection versus conventional in-vitro fertilization: first results. Hum Reprod. 1995;10(11):2835-9.

22. Hansen $M$, Kurinczuk JJ. et.al. The risk of major birth defects after intracytoplasmic sperm injection and in vitro fertilization. N Engl J Med. 2002;346(10):725-30.

23. Kacerovsky M, Musilova I, Hornychova $\mathrm{H}$, et al. Bedside assessment of amniotic fluid interleukin-6 in preterm prelabor rupture of membranes. Am J Obstet Gynecol. 2014;211(4):385. .e1-385.e3859.

24. Alger LS, Lovchik JC, Hebel JR, Blackmon LR, Crenshaw MC. The association of Chlamydia trachomatis, Neisseria gonorrhoeae, and group B streptococci with preterm rupture of the membranes and pregnancy outcome. Am J Obstet Gynecol. 1988;159(2):397-404.

\section{Figures}




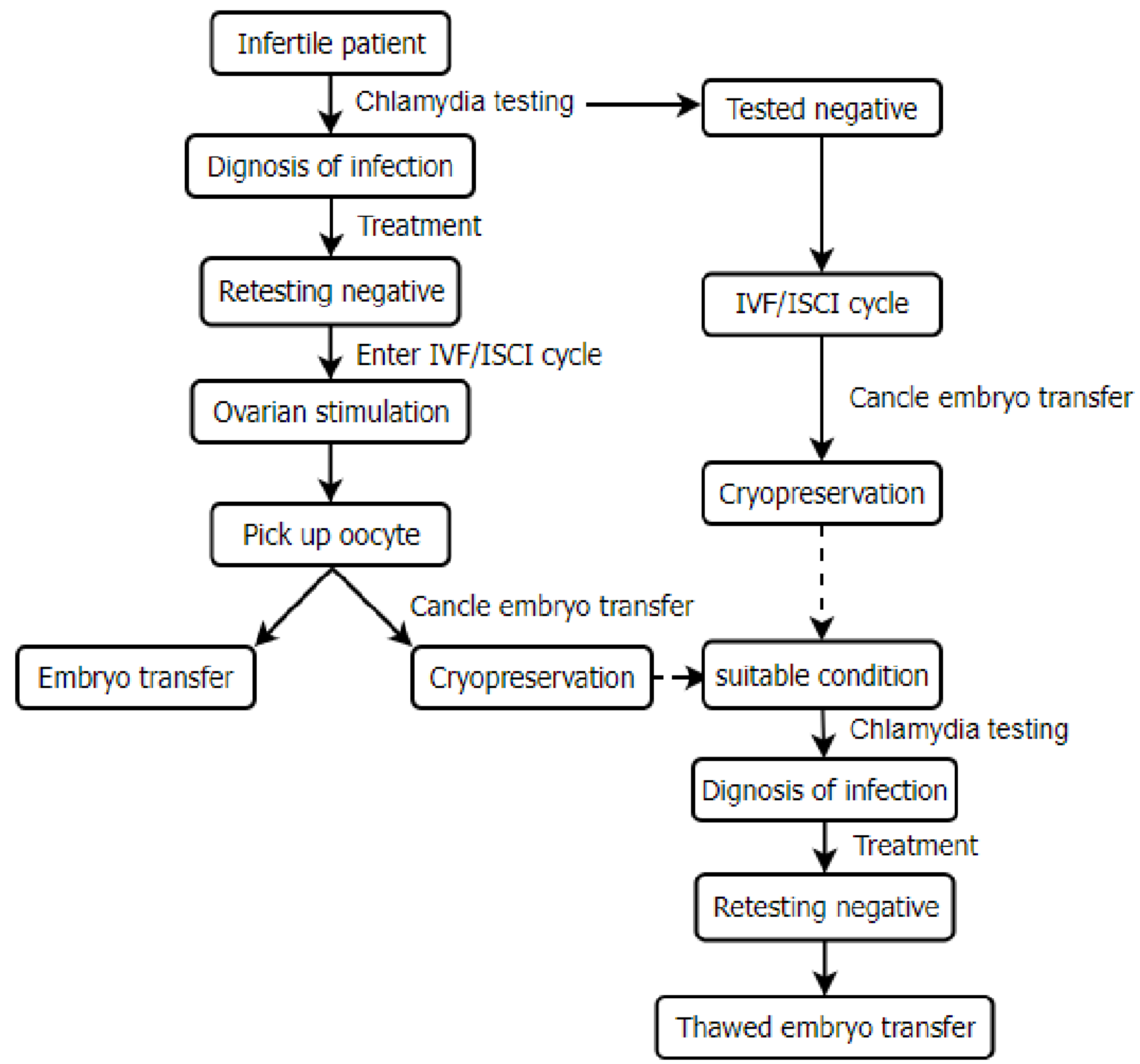

Figure 1

Timeline of Diagnosis and IVC/ICSI Stage of the Infected Group 


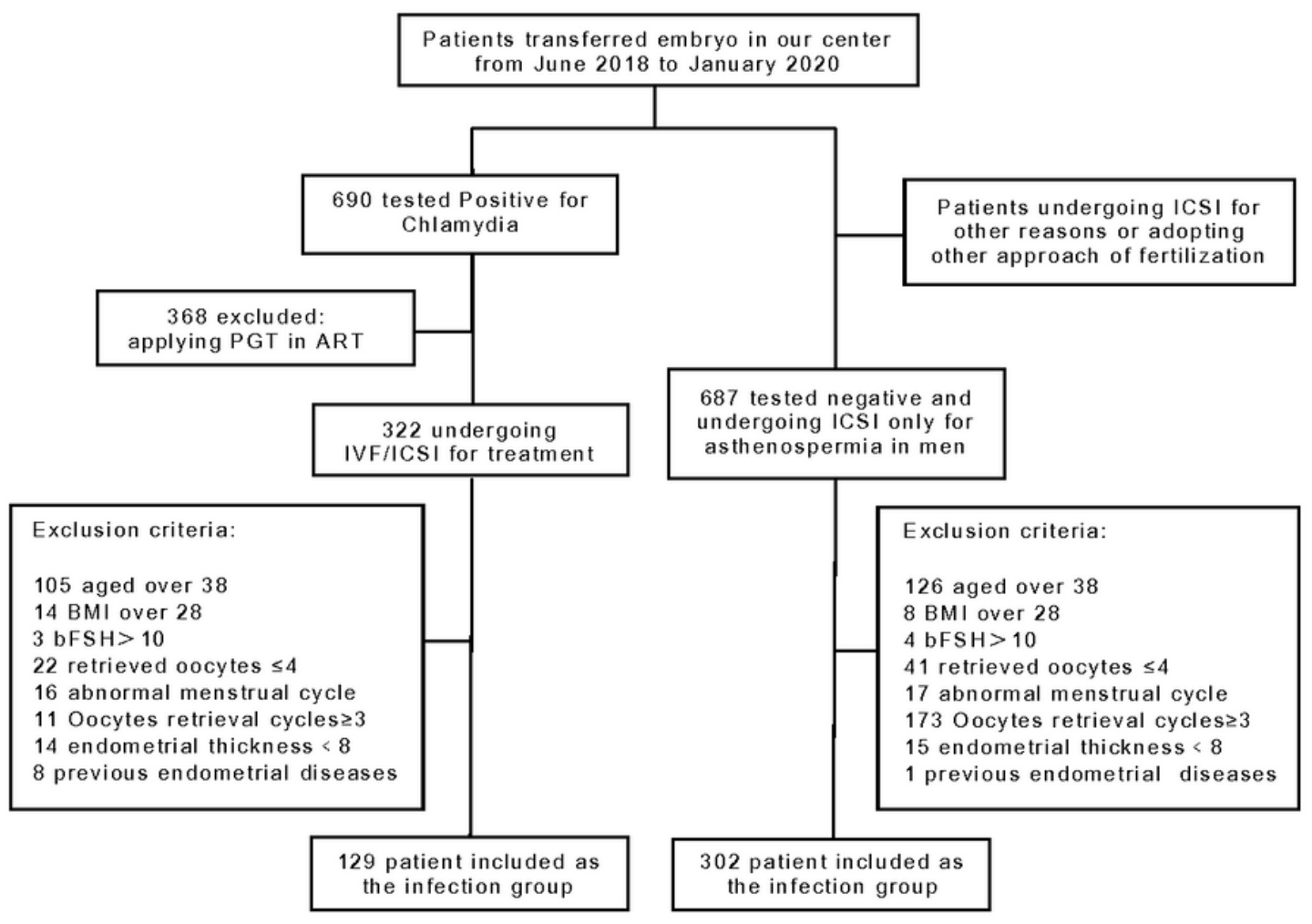

Figure 2

The recruitment process 